\title{
Routing Protocol for Mobile Nodes in Wireless Sensor Network
}

\author{
Bhagyashri Bansode \\ Department of Computer Engineering, Pune \\ Institute of Computer Technology, Pune, \\ Maharashtra, India.
}

\author{
Rajesh Ingle \\ Phd, Department of Computer Engineering, Pune \\ Institute of Computer Technology, Pune, \\ Maharashtra, India
}

\begin{abstract}
Wireless sensor network made up of sensor nodes which are fix or mobile. LEACH is clustered based protocol uses time division multiple access. It supports mobile nodes in WSN. Mobile node changes cluster. LEACH wait for two TDMA cycles to update the cluster, within these two cycles mobile node which changed cluster head, can't send data to any other cluster head, it causes packet loss. We propose an adaptive Low Packet Loss Routing protocol which support mobile node with low packet loss. This protocol uses time division multiple access scheduling to reserve the battery of sensor node. We form clusters, each cluster head update cluster after every TDMA cycle to reduce packet loss. The proposed protocol sends data to cluster heads in an efficient manner based on received signal strength. The performance of proposed LPLR protocol is evaluated using NS2.34 on Linux 2.6.23.1.42.fc8 platform. It has been observed that the proposed protocol reduces the packet loss compared to LEACH-Mobile protocol.

Keywords

Cluster based routing, mobility, LEACH-Mobile, WSN
\end{abstract}

\section{INTRODUCTION}

A wireless sensor network (WSN) consists of spatially distributed autonomous sensors to monitor physical or environmental conditions, such as temperature, sound, vibration, pressure, humidity, motion or pollutants and to cooperatively pass their data through the network to a main location. Modern networks are bi-directional, also enabling control of sensor activity. The development of wireless sensor networks was motivated by military applications such as battlefield surveillance; today such networks are used in many industrial and consumer applications, such as industrial process monitoring and control, machine health monitoring. WSN consist of mobile or fix sensor nodes. In some cases it consists of hybrid sensor nodes. All nodes sense and send data to server. This increases communication overhead because all nodes are sending data to server. This network containing hundreds or thousands of sensor node and main challenge in WSN is to reduce energy consumption and low packet loss in each sensor node. There are many routing protocols like Destination Sequenced Distance Vector (DSDV), Dynamic Source Routing (DSR), and Ad hoc On Demand Distance Vector (AODV) [1]. These protocols are supported to WSN but they are not suitable for tiny, low capacity sensor nodes and they require high power consumption. Flat-based multi-hop routing protocols, designed for static WSN [2-6], have also been exploited in WSN mobile nodes. However it not supports to mobility of sensor node
The main challenge in WSN is to minimize energy consumption in each sensor node. Many researchers concentrate on the routing protocol that would consume less power and hence prolong network's life span. Wireless ad hoc network routing protocols have been proposed for routing protocols in WSN.

Low Energy Adaptive Clustering Hierarchy-Mobile (LEACH-Mobile) [7] is routing protocol which support to WSN which have mobile nodes. LEACH-Mobile supports sensor nodes mobility in WSN by adding membership declaration to LEACH protocol. LEACH-Mobile protocol selects heads randomly and form cluster. Cluster head create Time Division Multiple Access (TDMA) schedule. Nodes sense and send that data to cluster head according to TDMA schedule. Mobility of node is big challenge to maintain cluster. Mobile nodes changes cluster continuously. LEACHMobile protocol update cluster after every two cycles of TDMA schedule. Packet loss happened in between two cycles of TDMA schedule. Mobile node which is not near to any cluster cannot send data to any cluster head so it causes packet loss.

Sensor nodes in LEACH-Mobile wait for two consecutive failure TDMA cycles, then cluster head decide that it has moved out of its cluster. During these two TDMA cycles sensor node loss the packets. In LPLR, sensor node does not need to wait for two consecutive TDMA cycles from cluster head to make decision. Cluster head directly decides that member node has moved out of its cluster after one TDMA cycle. The data loss is reduced by sending its data to new cluster head and sends join acknowledgment message to the cluster head.

We proposed a new low packet loss technique with efficient power consumption routing protocol for WSN. This proposed routing protocol called Low Packet Loss Routing Protocol for mobile nodes in wireless sensor network (LPLR Mobile-WSN). In our proposed protocol, the cluster head sends data request message to its members. When the cluster head does not receive data from its members, the packet is considered lost and cluster head delete membership of sensor node from the cluster. On the other hand, when the sensor node does not receive data request message from cluster head it will try to get entry into new cluster to avoid packet loss. Cluster head gives entry in TDMA schedule to incoming nodes from other cluster. Transmitter will send the message according to the received signal strength of data request message from the cluster head. 
Table 1 Abbreviations

\begin{tabular}{|l|l|}
\hline WSN & Wireless Sensor Network \\
\hline DSDV & Destination Sequenced Distance Vector \\
\hline DSR & Dynamic Source Routing \\
\hline AODV & $\begin{array}{l}\text { Low Energy Adaptive Clustering } \\
\text { Hierarchy-Mobile }\end{array}$ \\
\hline LEACH-Mobile & Time Division Multiple Access \\
\hline TDMA & Low Packet Loss Routing \\
\hline LPLR & Carrier Sense Multiple Access \\
\hline CSMA & $\begin{array}{l}\text { Collision Avoidance Medium Access } \\
\text { Control }\end{array}$ \\
\hline CA MAC & Cluster Head \\
\hline CH & Sensor Node \\
\hline SN & \\
\hline
\end{tabular}

\section{LOW PACKET LOSS ROUTING}

\section{Low Packet Loss Routing (LPLR) is Low Packet Loss} Routing protocol for wireless sensor network. LPLR proposes to handle packet loss and efficiently use energy resources. In this protocol, cluster head receives the data not only from its members during TDMA allocated time slot but also from other lost sensor nodes. WSN consist of mobile and fix both type of nodes. After cluster formation mobile node can change cluster. LPLR gives entry to mobile node in new cluster and TDMA schedule to send data.

\subsection{Selection of Cluster Head}

Protocols like TEEN [8] and APTEEN [9] used stationary but dynamically changing cluster head. In some protocol where sensor nodes are mobile cluster head is selected according to mobility factor [10]. The node with the smallest mobility factor in each cluster is chosen as cluster head. In LEACHMobile cluster head assumed to be stationary and static in order to control mobility. In proposed protocol we elect cluster heads randomly. It assumed to be stationary and static through the rounds.

\subsection{Formation of Cluster}

After a cluster head has been selected, it broadcasts an advertisement messages to the rest of the sensor nodes in the network as in LEACH and LEACH-Mobile. For these advertisement messages, cluster heads use a Carrier Sense Multiple Access with Collision Avoidance Medium Access Control (CSMA/CA MAC) protocol. All cluster heads use the same transmit energy when transmitting advertisement messages. Sensor nodes must keep their receivers "ON" in order to receive the advertisement message from their cluster head. After sensor nodes have received advertisement messages from one or more cluster heads, sensor nodes compare the received signal strength for received advertisement messages, and decide the cluster to which it will belong. By assuming symmetric propagation channels, the sensor node selects cluster head to which the minimum amount of transmitted energy is needed for communication. In the case of a tie, a random cluster-head is chosen. After deciding the cluster it will belong, the node sends registration message to inform the cluster head. This advertisement messages are transmitted to the cluster heads using CSMA/CA MAC protocol. During this phase, all cluster heads must keep their receiver on.

\subsection{TDMA Schedule Creation}

After cluster head receives registration messages from the nodes that would like to join the cluster, the cluster head creates a TDMA schedule based on the number of nodes and assigns each node a time slot to transmit the data. This schedule is broadcasted to all the sensor nodes in the cluster. All sensor nodes will transmit data according to TDMA schedule

\subsection{Data Transmission}

Once the clusters are created and the TDMA schedule is fixed, data transmission from sensor nodes to their cluster heads begin according to their TDMA scheduled. Upon receiving data request from the cluster head the sensor node switches on its radio transmitter, adjusts transmission power and sends its data. At the end of the transmission, the node turns off its radio, thus we can save the battery of sensor node. The cluster head must keep its radio on to send data request messages, receive data from the sensor nodes and to send and receive other messages needed to maintain the network. Sensor node receives data request message from the cluster head, it will send its data back to the cluster head. If the sensor nodes did not receive data request message from its cluster head, it will send the message to a free cluster head.

\section{LPLR ALGORITHM}

Cluster Head $-\mathrm{CH}_{1}$ to $\mathrm{CH}_{6}$

Sensor node $-S_{1}$ to $S_{51}$

1. Select head randomly.

Select cluster head $\mathrm{CH}_{1}$ to $\mathrm{CH}_{6}$ from $\mathrm{S}_{1}$ to $\mathrm{S}_{51}$ sensor node.

2. All cluster head broadcast advertisement message, from $\mathrm{S}_{1}$ to $S_{51}$ except cluster heads.

3. Sensor node receives advertisement messages from $\mathrm{CH}_{1}$ to $\mathrm{CH}_{6}$.

4. Compare received signal strength. Select maximum received signal strength. According to that received signal strength sensor node select head $\mathrm{CH}_{1}$ to $\mathrm{CH}_{6}$.

5. Sensor node sends registration message to cluster head.

6. Cluster head create TDMA schedule and broadcast to all member nodes.

7. According to TDMA schedule cluster head sends data request message to member node.

8. Member node sends data to cluster head.

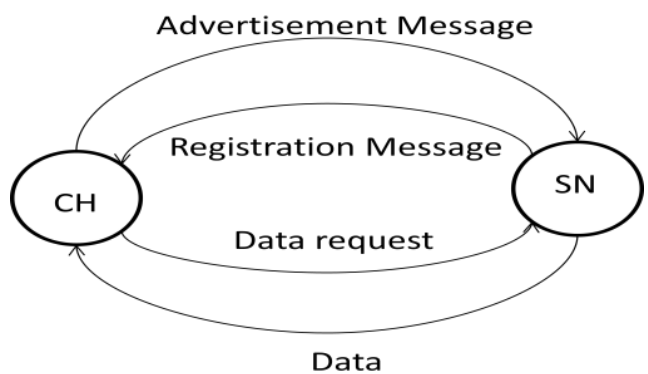

$\mathrm{CH}$-Cluster Head

SN-Sensor Node

Figure 1: Messages of Cluster Head and Sensor Node

\subsection{Cluster Head}

Cluster head broadcast advertisement message. If cluster head receive registration message then it create TDMA schedule 
and broadcast that schedule. When cluster head finishes receiving data messages from all sensor nodes, it will check whether it receives data messages from all members. Any of the member nodes did not send the data message then cluster head remove that sensor node from cluster. Cluster head again broadcast advertisement message to all nodes and update the cluster. Updating of cluster causes entry of new mobile nodes in TDMA schedule of cluster.Figure1 shows messages transfers and receives by cluster head.

\subsection{Sensor Node}

Sensor node receives advertisement message from one or more cluster head. According to received signal strength node select head. Node send registration message to selected head and get entry into TDMA schedule of that cluster. Member node sends data to cluster head according to TDMA schedule when it receive data request message from cluster head. If sensor node does not receive any advertisement or any data request message then it sends data to free cluster head. Figure1 shows messages transfers and receives by sensor node.

\section{SIMULATION RESULT}

We have simulated LPLR and LEACH-Mobile using NS2.34 on Linux 2.2.23.1.42.fc8 platform with parameters as shown in Table 1. Basic hardware requirement for this simulation is pentium4 processor, 512mb RAM, 10GB hard disk.

\section{Table 2 Performance Parameter}

\begin{tabular}{|l|l|}
\hline Parameter & Value \\
\hline Network Size $\left(\mathrm{L}^{*} \mathrm{~W}\right)$ & $1800 * 840$ \\
\hline Number of Sensor Nodes $(\mathrm{N})$ & 51 \\
\hline Percentage of Cluster Head & $10 \%$ \\
\hline $\begin{array}{l}\text { Percentage of Mobile Sensor } \\
\text { Node }\end{array}$ & $90 \%$ \\
\hline Sensing Range & $500 \mathrm{~m}$ \\
\hline Sensor speed & $1-17 \mathrm{~m} / \mathrm{s}$ \\
\hline
\end{tabular}

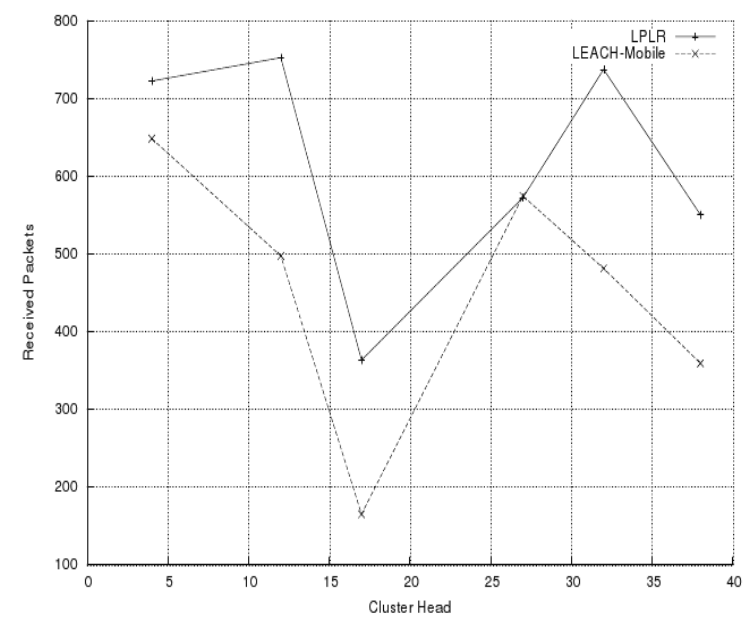

Figure2. Total Number of Received Packets

Figure2. shows total number of packets received by cluster head from member node. We applied LEACH-Mobile to network and observed number of packets received by each cluster head from member node in the WSN. We applied LPLR to the same WSN and observed same result. We can see from figure 2 the LPLR achieves significant improvement compared to LEACH-Mobile. We can conclude that packet loss decreases using LPLR

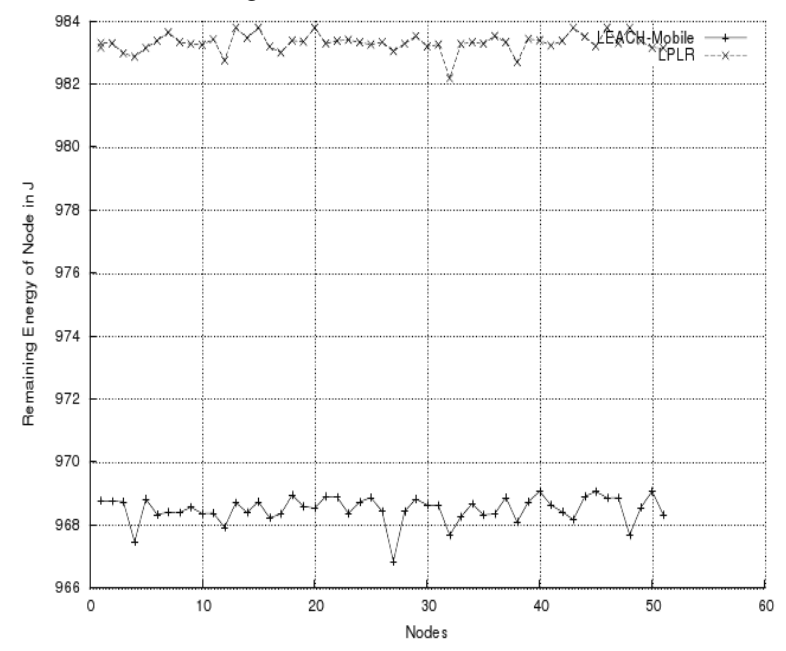

Figure3. Remaining Energy of Nodes

Figure 3 shows remaining energy of every sensor node in the WSN. Member node wakes up to send the data according to TDMA schedule otherwise they are in sleep mode so we can reduce energy consumption. We can compare the remaining energy of sensor node in LEACH-Mobile and LPLR from figure 3. We can say that sensor node can reserve more battery using LPLR than LEACH-Mobiles.

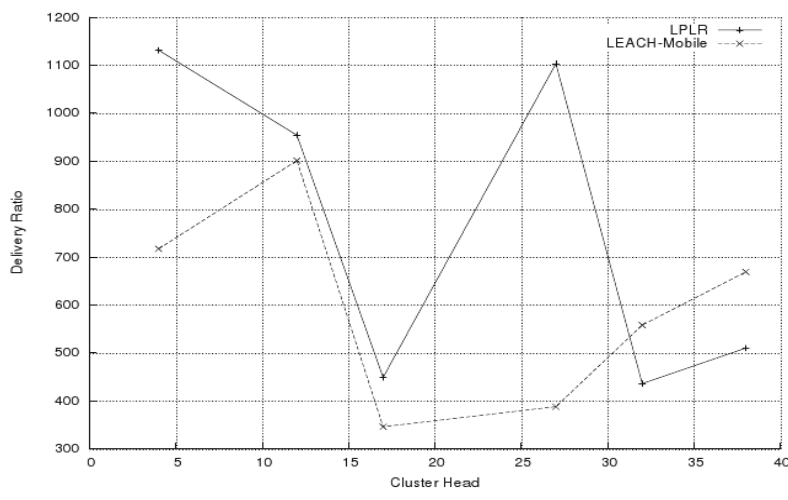

Figure4. Packet Delivery Ratio

Figure4 shows packet delivery ration of cluster head of LPLR and LEACH-Mobile. From this figure we can compare delivery ratio of both protocol and performance of LPLR is efficient than LEACH-Mobile.

\section{CONCLUSION}

We proposed cluster based routing protocol LPLR which is efficient as compare to LEACH-Mobile protocol. In proposed protocol all sensor nodes are maintain by cluster head. TDMA schedule is also maintained by cluster head. Cluster head collect data from member node and sends that data to server. If sensor node fails or battery of node is get discharged then cluster head remove record of that node from TDMA schedule and update schedule. Cluster head failure can affect the working of WSN. Member node cannot send data to cluster head in case of cluster head failure. It causes packet loss. We 
are working on cluster head failure case to get better result than proposed LPLR protocol.

We proposed efficient routing protocol for mobile nodes in wireless sensor network. LPLR protocol is efficient in energy consumption and packet delivery in the network. It forms cluster. Cluster head create and maintain TDMA schedule. Sensor node wake up at the time of sending data and it goes into sleep mode, it reserve the battery of sensor node. Important feature of this protocol is it update cluster after every TDMA cycle. So every mobile node can get entry into new cluster and can send data. LPLR also maintain a free cluster head. Mobile sensor node which is not in the range of any cluster or node which are moved from one cluster and waiting for new cluster they sends data to free cluster head. It reduces packet loss. We have simulated LEACH-Mobile and proposed LPLR protocol and got efficient result of LPLR.

\section{REFFERENCES}

[1] C.Perkins and P.Bhagwat. "Highly Dynamic DestinationSequenced Distance-Vector Routing (DSDV) for Mobile Computers," presented at the ACM '94 Conference on Communications Architectures, Protocols and Applications, 1994.

[2]W. Heinzelman, J. Kulik, and H. Balakrishnan, "Adaptive protocols for information dissemination in wireless sensor networks," Proc. 5th ACM/IEEE Mobicom Conference (MobiCom '99), Seattle, WA, August, 1999, pp. $174-85$

[3]J.Kulik, W. R. Heinzelman, and H. Balakrishnan, "Negotiation-based protocols for disseminating information in wireless sensor networks," Wireless Networks, Vol. 8, 2002, pp. 169-185.
[4] C. Intanagonwiwat, R. Govindan, and D. Estrin, "Directed diffusion: a scalable and robust communication paradigm for sensor networks," Proc. of ACM MobiCom '00, Boston, MA, 2000, pp. 56-67.

[5] D. Braginsky and D. Estrin, "Rumor routing algorithm for sensor networks," Proc. of the 1st Workshop on Sensor Networks and Applications (WSNA), Atlanta, GA October 2002.

[6]Y. Yao and J. Gehrke, "The cougar approach to in-network query processing in sensor networks", in SIGMOD Record, September 2002.

[7]Guofeng Hou, K. Wendy Tang, "Evaluation of LEACH protocol Subject to Different Traffic Models," presented at the first International conference on Next Generation Network (NGNCON 2006), Hyatt Regency Jeju, Korea/July 9-13,1006

[8]A. Manjeshwar and D. P. Agarwal, "TEEN: a routing protocol for enhanced efficiency in wireless sensor networks," presented at the 1st Int. Workshop on Parallel and Distributed Computing Issues in Wireless Networks and Mobile Computing, April 2001

[9]A. Manjeshwar and D. P. Agarwal, "APTEEN: A hybrid protocol for efficient routing and comprehensive information retrieval in wireless sensor networks," Parallel and Distributed Processing Symposium., IPDPS 2002, pp. 195-202.

[10]M. Liliana, C. Arboleda, and N. Nasser, "Cluster-based Routing Protocol or Mobile Sensor Networks," presented at the 3rd Int. Conf. on Quality of Service in Heterogeneous Wired/Wireless Networks, Waterloo, Ontario, Canada, 2006 